\title{
OUABAIN - A Drug for Treatment of COVID-19
}

\author{
Knut Sroka* \\ Vizelinstrasse 58, 22529 Hamburg, Germany \\ ${ }^{\star}$ Corresponding author: Knut Sroka, Hamburg, Germany; E-Mail: dr.sroka@gmx.de
}

Received: June 28, 2020; Accepted: July 06, 2020; Published: July 15, 2020

\begin{abstract}
There is an enormous demand for effective medication against COVID-19. The cardiac steroid ouabain has properties qualifying it as a drug in the battle against the actual corona pandemic. Strong antiviral properties against different viruses including SARS- CoV-2 have been circumstantiated. Ouabain increases the resistance to hypoxia and exerts protecting effects in cytokine dysregulation. The application of ouabain could to be useful at any stage of COVID-19.
\end{abstract}

Keywords: Antiviral, ARS-CoV-2, COVID-19, Cytokine dysregulation, Ouabain

\section{Introduction}

SARS-CoV-2 has locked down the world. Human victims and the economic damage are tremendous. The need for effective medication in COVID-19 is immense. Ouabain is a drug extracted from the seeds of an African vine. The favorable effects of this drug in different forms of heart disease have been well known for longtime. Nowadays ouabain is used in cancer and antiviral research. In this article the antiviral properties of ouabain and its potential effects in treating COVID-19 patients are presented.

\section{Specific Qualities of Ouabain}

\section{Strong Antiviral Properties}

Ouabain, a cadiotonic steroid, is part of the cardioactive glycosides. Ouabain has strong antiviral properties. Three examples: (a) The replication of influenza A virus will be inhibited almost completely by ouabain. $24 \mathrm{~h}$ post-infection with influenza $A$ the viral titers were found to be decreased by $99.1 \%$ with ouabain treatment [1]. (b) Ouabain was shown to reduce the replication rate of Ebola virus by $50 \%$ within 48 $\mathrm{h}$ [2]. (c) Ouabain was identified as having robust efficiency against Japanese Encephalitis Virus (JEV) infection. JEV infection was blocked by ouabain at the replication stage within $24 \mathrm{~h}$. Furthermore, it was proven that ouabain significantly reduced the morbidity and mortality caused by JEV in a BALB/c mouse model [3]. The ability of ouabain to inhibit virus replication has been proven for different other viruses with a lipid envelope: Herpes simplex virus, vaccinia poxvirus, murine leukemia virus, SARS corona viruses, and others.

In the vast majority of publications these antiviral effects of ouabain are interpreted as the result of the inhibition of the sodium pump (NKA) by cardiac glycosides. This interpretation is wrong. It ignores the ouabain-research within the last decades. In the nineties ouabain was identified as an endogenous hormone. This discovery led to an intense re-examination of the drug. Till today, the existence of an endogenous ouabain produced in the adrenal cortex is still discussed controversially. In the course of the following research activities it was discovered that ouabain in nanomola concentrations stimulates the cardiac sodium pump [4]. The inhibitory effect on cardiac NKA needs millimolar concentrations. Ouabain has hormetic properties. That means a low dose leads to stimulation or beneficial effects and a high dose to inhibitory or toxic effects.

Today it is well-documented that ouabain in low concentration can activate multiple signal transduction pathways [5]. Ouabain binds to a region of the NKA which is located in caveolae on the sarcolemma. The caveolae contain signalosomes which form a vesicular signaling platform. When ouabain binds to NKA different proteins complexes are activated. They send signals to the cell, to intracellular signaling complexes, to mitochondria, and the nucleus. The activated signal cascades by ouabain induce homeostatic and protective effects. The signaling activities of ouabain occur without affecting the ion pumping function of the NKA [6]. The strong effects of ouabain on virus replication need a low concentration of the drug. Concentration of $20 \mathrm{nMol}$ was successful in tissue cultures with influenza A and Ebola virus. In this concentration ouabain exerts a stimulating effect on the sodium pumps. Recently it was shown that ouabain suppresses coronaviral replication. The authors of this study did not restrict their view on activation or inhibition of the NKA. They looked for potential signal pathways involved in this antiviral effect. They showed that ouabain suppresses coronaviral replication via amplifying a signal cascade in the cytoplasm. Augmenting the NKA-dependent PI3K_ PDK1 axis signaling by ouabain contributed essentially to antiviral activity and replication [7].

Just published is a study from Korea which aimed to assess antiviral activity of ouabain and digoxin against SARS-CoV-2 infection [8]. The half-maximal inhibitory concentration (IC50) of ouabain and digoxin were determined at a nanomolar concentration. Virus titers of single dose treatment of ouabain and digoxin showed a $>99 \%$ inhibition of SARS-CoV-2 replication. In comparison with chloroquine, ouabain and digoxin were much more effective. Notably, ouabain and digoxin 
significantly inhibited viral mRNA expression, copy number and viral protein expression when administered at the post-entry stage. When given at the host entry stage of the virus cycle digoxin did not show effective antiviral activity. However, ouabain treatment at the entry stage inhibited approximately $50 \%$ of viral mRNA expression and protein expression.

In research and clinics both cardiac glycosides - ouabain and digitalis derivates - are equalized with regards to their mode of action and their clinical effects. There is a need for differentiation [9]. The hydrophilic ouabain acts at the surface of the cells. The lipophilic digitalis glycosides penetrate the cell membrane and interact with complete different receptors in the cytoplasm. There are important differences in signal transduction and clinical effects between ouabain and digitalis glycosides.

\section{Increasing the Resistance to Hypoxia}

In COVID-19 lack of oxygen is crucial. In dogs, application of Ouabain increases the resistance to hypoxia [10]. Seventy years ago already the German physiologist Rein reported that on treatment with ouabain "the animal simply became resistant against oxygen deficiency for hours" [10]. Recently, it was shown that ouabain, given immediately before an anoxic period of 30 minutes, protected rat hearts as documented by an improved recovery of contractile function and a reduction of infarct size [11]. The interaction of ouabain with the $\mathrm{Na}+\mathrm{K}+-\mathrm{ATPase}$ activates a cellular signaling cascade involving src kinase, mitoKATP, and ROS. Via this pathway ouabain protects the outer mitochondrial membrane integrity, adenine nucleotide compartmentation, and energy transfer efficiency. By preserving the mitochondrial function ouabain boosts the resistance of the myocardium against oxygen deficiency.

\section{Protection against Cytokine Dysregulation}

Cytokine dysregulation is a common feature in COVID-19. A dysregulated immune response to SARS-CoV-2 appears to drive mortality in this disease. A subset of COVID-19 patients is characterized by the development of a cytokine storm syndrome (CSS). Interleukin (IL)-6 levels, and tumor necrosis factor (TNF)- $\alpha$ levels are predictors of COVID-19 severity and in-hospital mortality. Targeting cytokine dysregulation in COVID-19 could be critical for reducing mortality.

Ouabain protects against cytokine dysregulation. It acts in different ways, (a) by interacting directly with immunocytes and (b) by stimulation of vagal activity.

(a). To illustrate the interaction of ouabain with immunocytes, the following paper will be quoted. In the study: "Modulation of Cytokine Production and Protection against Lethal Endotoxemia by the Cardiac Glycoside Ouabain" [12] human peripheral blood mononuclear cells (PBMC) were obtained from healthy volunteers. PBMC were cultured with or without ouabain in the presence or absence of lipopolysaccharide (LPS). LPS stimulates immunocytes, mainly macrophages, to generate IL-1, IL-6, TNF- $\alpha$, and other substances. When PBMC were stimulated with LPS, ouabain suppressed the production of IL-6 and TNF- $\alpha$. To investigate whether ouabain modulates cytokine production in vivo, the effects of ouabain in LPS-treated mice were evaluated. Ouabain was found to protect against LPS-induced lethal toxicity in mice and decreased circulating IL-6 and TNF- $\alpha$ levels in vivo [12].

(b). It is becoming increasingly clear that the autonomic nervous system and the immune system demonstrate cross-talk during inflammation by means of sympathetic and parasympathetic pathways. On the parasympathetic side, a vagus nerve-mediated inflammatory reflex was recognized during the past years. The central nervous system recognizes peripheral inflammation via afferent vagus nerve signaling. The efferent arm of this reflex is referred to as the "cholinergic anti-inflammatory pathway". Vagal nerve signaling to macrophages in the spleen inhibit proinflammatory cytokine production and attenuate peripheral innate immune response. Stimulation of the vagus nerve prevents the damaging effects of cytokine release in experimental sepsis, endotoxemia, ischemia/reperfusion injury and other inflammatory syndromes [13-15].

Cholinergic agonists inhibit pro-inflammatory cytokines synthesis and prevent cytokine-mediated diseases [13]. Ouabain is a strong cholinergic agonist by inducing the release of acetylcholine from the peripheral postganglionic vagal fibers. This qualifies ouabain as an agent to protect effectively against cytokine dysregulation. In a cytokine storm syndrome, catecholamines are involved and make the cytokine dysregulation worse. Ouabain antagonizes the effects of catecholamines. It was shown that ouabain diminishes severely the nor-adrenaline content of the blood in heart insufficiency patients [16]. Low concentrations of ouabain are able to block the adrenaline release from adrenal medulla [17]. Sympathetic over activity and catecholamines enhance inflammatory injury by augmenting the production of IL- 6 and other cytokines. Vagal activity and ouabain will protect against a cytokine storm.

\section{Conclusion}

Three specific features of ouabain are highlighted: Ouabain has strong antiviral capabilities against different viruses including SARS$\mathrm{CoV}-2$ in vitro. Application of Ouabain in vivo increases resistance to hypoxia and exerts protective effects against cytokine dysregulation. This all together qualifies ouabain as a valuable drug in the treatment of COVID-19.

Many COVID-19 patients have heart problems, high blood pressure, coronary artery disease or heart insufficiency. Exactly for these diseases ouabain was the leading medicine in Europe and particular in Germany for decades in the last century. Then it was marginalized by the introduction of beta blockers and ACE inhibitors in the nineties. Clinicians today regard Ouabain as contraindicated for coronary patients. The general belief is: Cardiac steroids block the sodium pump leading to calcium influx, inotropy and an augmentation of cardiac oxygen consumption which is deleterious for these patients. This reasoning neglects the hormetic character of ouabain. It ignores the multiple signaling effects activated by Ouabain. It overlooks that Ouabain in low concentration diminishes cardiac 
oxygen consumption [18]. In COVID-19 patients with heart problems Ouabain is particularly suitable.

In COVID-19 ouabain should be given at any stage of the disease. Symptoms plus positive testing would be the best starting point to prevent serious complications. Intensive care physicians should gain experience with ouabain in cases of a severe course of the disease. The current study from Korea [8] shows strong effects of ouabain at the host entry stage of the virus cycle. As a large number of patients are asymptomatic at the initial stage of SARS-CoV-2 infection, it could be useful to take ouabain prophylactically. This could prevent an increase in disease incidence, especially in slum areas and favelas.

Ouabain is used in Germany as an oral application - tablets and liquids - and as an intravenous injection. These medicines are natural products of the strophanthus vine. Ouabain can be synthesized chemically. Upon daily i.v.-administration of $0.5 \mathrm{mg}$ ouabain to normal subjects, a steady-state plasma concentration of $0.5 \mathrm{ng} / \mathrm{ml}$ has been determined [19]. A median therapeutic dose is $0.125-0.25 \mathrm{mg}$ Ouabain i.v. twice per day. In this dosage intravenous Ouabain is safe and free of any side effects. Orally taken, the effect levels of ouabain are in the same low nanomolar range. Oral Ouabian is also effective and free of side effects. Hypokalaemia and hypercalcemia are contraindications, as well as bradycardic rhythm and conduction disorders.

My respect and much gratitude to Dr. Hauke Fürstenwerth for his extensive scientific activity on the topic of ouabain.

\section{References}

1. Hoffmann H-H, Palese P, Shaw ML (2008) Modulation of influenza virus replication by alteration of sodium ion transport and protein kinase $\mathrm{C}$ activity. Antiviral Res 80 : 124-134. [crossref]

2. Garcia-Dorival I, Wu W, Dowall S, Stuart Armstrong, Olivier Touzelet, et al. (2014) Elucidation of the Ebola Virus VP24 Cellular Interactome and Disruption of Virus Biology Through Targeted Inhibition of Host-Cell Protein Function. J Proteome Res 13: 5120-5135. [crossref]

3. Guo J, Jia X, Liu Y, Wang S, Junyuan Cao 1 2, Bo Zhang, et al. (2020) Screening of Natural Extracts for Inhibitors against Japanese Encephalitis Virus Infection. Antimicrob Agents Chemother 64: e02373-19. [crossref]

4. Gao J, Wymore RS, Wang Y, Glenn R. Gaudette, Irvin B. Krukenkamp, et al. (2002) Isoform-specific stimulation of cardiac $\mathrm{Na} / \mathrm{K}$ pumps by nanomolar concentrations of glycosides. J Gen Physiol 119: 297-312. [crossref]
5. Aperia A (2007) New roles for an old enzyme: Na,K-ATPase emerges as an interesting drug target. J Int Med 261: 44-52. [crossref]

6. Zhang L, Zhang Z, Guo H, Yongli Wang (2008) Na+/K+-ATPase-mediated signal transduction and $\mathrm{Na}+/ \mathrm{K}+-\mathrm{ATPase}$ regulation. Fundam Clin Pharmacol 22: 615-621. [crossref]

7. Yang CW, Chang HY, Lee YZ, Hsing-Yu Hsu, Shiow-Ju Lee (2018) The cardenolide ouabain suppresses coronaviral replication via augmenting a $\mathrm{Na}+\mathrm{K}+$-ATPasedependent PI3K_PDK1 axis signaling. Toxicology and Applied Pharmacology 356: 90-97. [crossref]

8. Cho J, Lee YJ, Kim J-H, Sang il Kim, Sung Soon Kim, et al. (2020) Antiviral activitiy of digoxin and ouabain against SARS-CoV-2 infection and its implication for COVID-19. DOI: https://doi.org/10.21203/rs.3.rs-34731/v1

9. Fuerstenwerth H (2011) On the Differences Between Ouabain and Digitalis Glycosides. Am J Ther 21: 35-42. [crossref]

10. Rein H (1949) Über ein Regulationssystem Milz-Leber für den oxidativen Stoffwechsel der Körpergewebe und besonders des Herzens. Naturwissenschaften 36 233-239, 36: 260-268, (quoted from Fuerstenwerth H (2012) Rethinking heart failure. Cardiol Res 3: 243-257.

11. Pasdois P, Quinlan CL, Rissa A, Liliane Tariosse, Béatrice Vinassa, et al. (2007) Ouabain protects rat hearts against ischemia-reperfusion injury via pathway involving src kinase, mitoKATP, and ROS. Am J Physiol Heart Circ Physiol 292: H1470-1478. [crossref]

12. Matsumori A, Ono K, Nishio R, Igata H, Shioi T, et al. (1997) Modulation of Cytokine Production and Protection Against Lethal Endotoxemia by the Cardiac Glycoside Ouabain. Circulation 96: 1501-1506. [crossref]

13. Tracey KJ (2007) Physiology and immunology of the cholinergic antiinflammatory pathway. J Clin Invest 117: 289-296. [crossref]

14. Huston JM (2012) The vagus nerve and the inflammatory reflex: wandering on a new treatment paradigm for systemic inflammation and sepsis. Surg Infect (Larchmt) 13: 187-193. [crossref]

15. Boeckxstaens $\mathrm{G}$ (2013) The clinical importance of the anti-inflammatory vagovaga reflex. Handb Clin Neurol 117: 119-134. [crossref]

16. Agostini PG Doria E, Berti M, Guazzi MD (1994) Long-term use of k-strophanthin in advanced congestive heart failure due to dilated cardiomyopathy: a double-blind crossover evaluation versus digoxin. Clin Cardiol 17: 536-554. [crossref]

17. Gutman Y, Boonyaviroj P (1977) Mechanism of inhibition of catecholamine release from adrenal medulla by diphenylhydantoin and by low concentrations of ouabain (10(- 10)M). Naunyn Schmiedebergs Arch Pharmacol 296: 293-296. [crossref]

18. Sroka K (2015) Ouabain: a re-evaluation. Med Welt 66: 275-280 (in German).

19. Selden R, Smith TW, Findley W (1972) Ouabain Pharmacokinetics in Dog and Man. Circulation 45: 1176-1182. [crossref]

\section{Citation:}

